\title{
Pulsenet - A Parallel Flash Sampler and Digital Processor IC for Optical SETI
}

\author{
Andrew W. Howard ${ }^{1}$, Gu-Yeon Wei ${ }^{1}$, William J. Dally ${ }^{2}$, and Paul Horowitz ${ }^{1}$ \\ ${ }^{1}$ Harvard University, Cambridge, MA \\ ${ }^{2}$ Stanford University, Stanford, CA
}

\begin{abstract}
PulseNet is a full-custom IC with parallel flash ADC and digital processing that enables an all-sky optical search for extraterrestrial intelligence. It integrates 448 sense amplifiers that digitize 32 analog signals at $1 \mathrm{GS} / \mathrm{s}$, and other circuits that filter samples, store candidate signals, and perform astronomical observations. Its $\sim 250,000$ CMOS transistors (TSMC $0.25 \mu \mathrm{m}$ ) dissipate $1.1 \mathrm{~W}$ at $400 \mathrm{MHz}$ and $2.5 \mathrm{~V}$.
\end{abstract}

\section{INTRODUCTION}

PulseNet is a full-custom flash ADC and digital processor chip designed for an all-sky optical search for extraterrestrial intelligence (SETI). It digitizes 32 analog signals at up to $1 \mathrm{GS} / \mathrm{s}$, and filters in real-time for intentional signals from other advanced civilizations. Thirty-two of these ICs form the computing core of our all-sky optical SETI instrument. Together they digitize and filter $\sim 3.5 \mathrm{~Tb} / \mathrm{s}$ - roughly the contents of all books in print, every second. The strength of PulseNet is not optimization of a single parameter; rather, it is the experiment-driven on-chip integration of several demanding electronic components into an instrument that would otherwise be impossible to construct.

Astronomical searches for signals from other civilizations have been performed primarily in the microwave radio spectrum (sources whose spectral width is too narrow for physical processes) and in the visible optical spectrum (pulses whose short duration and brightness are incompatible with known astrophysical objects). The scope of these searches is frequently limited by technology-in radio SETI, the FFTs performed by ever expanding arrays of FPGAs, DSPs, and PCs have greatly increased the spectral coverage and sensitivity. Optical SETI before PulseNet was limited to observing candidate stars one at a time ( $\sim 104$ stars and $\sim 10-5$ of the sky area observed in several years) [1]. With PulseNet, we can survey the entire Northern sky for optical signals in less than a year.

Optical SETI is based on this remarkable fact: using today's technology, we could generate an optical signal that would be visible across much of the galaxy, and that would be distinguishable from astrophysical phenomena and noise. For example, a Helios laser (4.7MJ pulses lasting 3ns) coupled into a $10 \mathrm{~m}$ Keck-class optical telescope would outshine the Sun by a factor of $\sim 10^{4}$ in broadband visible light during the brief pulse and in the narrow direction of the target. At a range of $10^{3}$ lightyears (within which there are $\sim 10^{6}$ Sun-like stars), the signal would deliver $\sim 1500$ optical photons to a similar $10 \mathrm{~m}$ telescope at the target [1]. Thus, optical communication between civilizations is plausible, and searches like ours are justified.

Our all-sky optical search scheme is shown schematically in Fig. 1. It uses a $\mathrm{f} / 2.51 .8 \mathrm{~m}$ optical telescope that images a $1.6^{\circ} \times 0.2^{\circ}$ patch of the sky on two identical arrays of photomultiplier tube (PMT) pixels through a beamsplitter. The telescope is fixed and the imaging beam drifts across the sky as the Earth rotates. To minimize noise, we require that a signal be seen coincidentally in a matched pair of pixels in the two PMT arrays. The PMTs are sensitive to single photons, which produce $\sim 3-5 \mathrm{~ns}$ voltage pulses $(\sim 10-50 \mathrm{mV}$, depending on gain), and are approximately linear for larger signals.

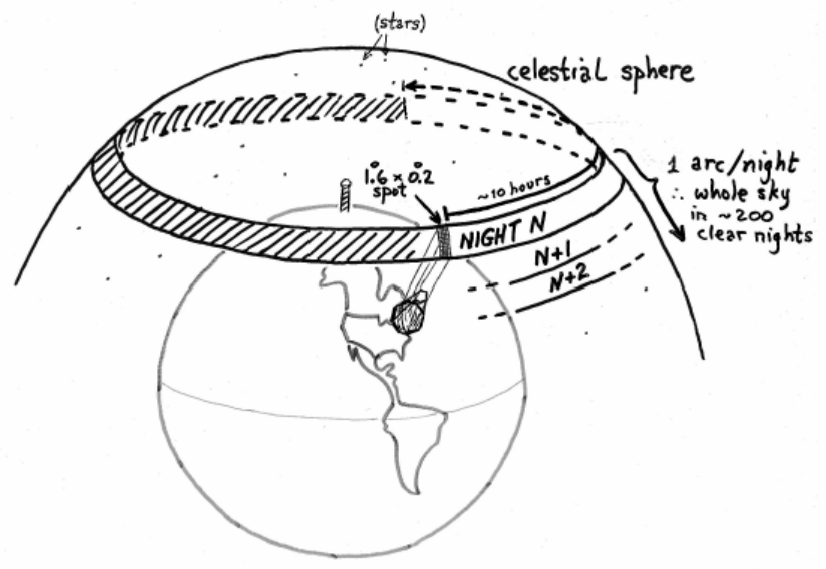

Figure 1. All-sky optical search scheme

\section{PUlseNet DesigN}

Each PulseNet handles analog signals from 32 PMT pixels (16 matched pairs) as shown in Fig. 2. These signals are digitized at $1 \mathrm{GS} / \mathrm{s}$ by comparing each to seven external voltages $\left(\mathrm{V}_{\mathrm{REF}}[6: 0]\right)$ on the rising edges of two interleaved clocks (clka/clkb; 500MHz). The resulting 7-bits/pixel/clock of thermometer code are encoded to 3-bits/pixel/clock and are delayed by an 8-bit deep array of 2-phase shift registers. Meanwhile, a coincidence trigger circuit selects one thermometer code bit from all 32 pixels (and both clocks) and looks for instances when a matched input pair simultaneously exceed a given threshold - a "coincidence", e.g., pixels $14 \mathrm{~A}$ and $14 \mathrm{~B}$ both exceed $\mathrm{V}_{\mathrm{REF}}[3]$ on a rising edge of clka. Coincidences trigger switches that steer streaming data from the input pair into 256-bit long shift 


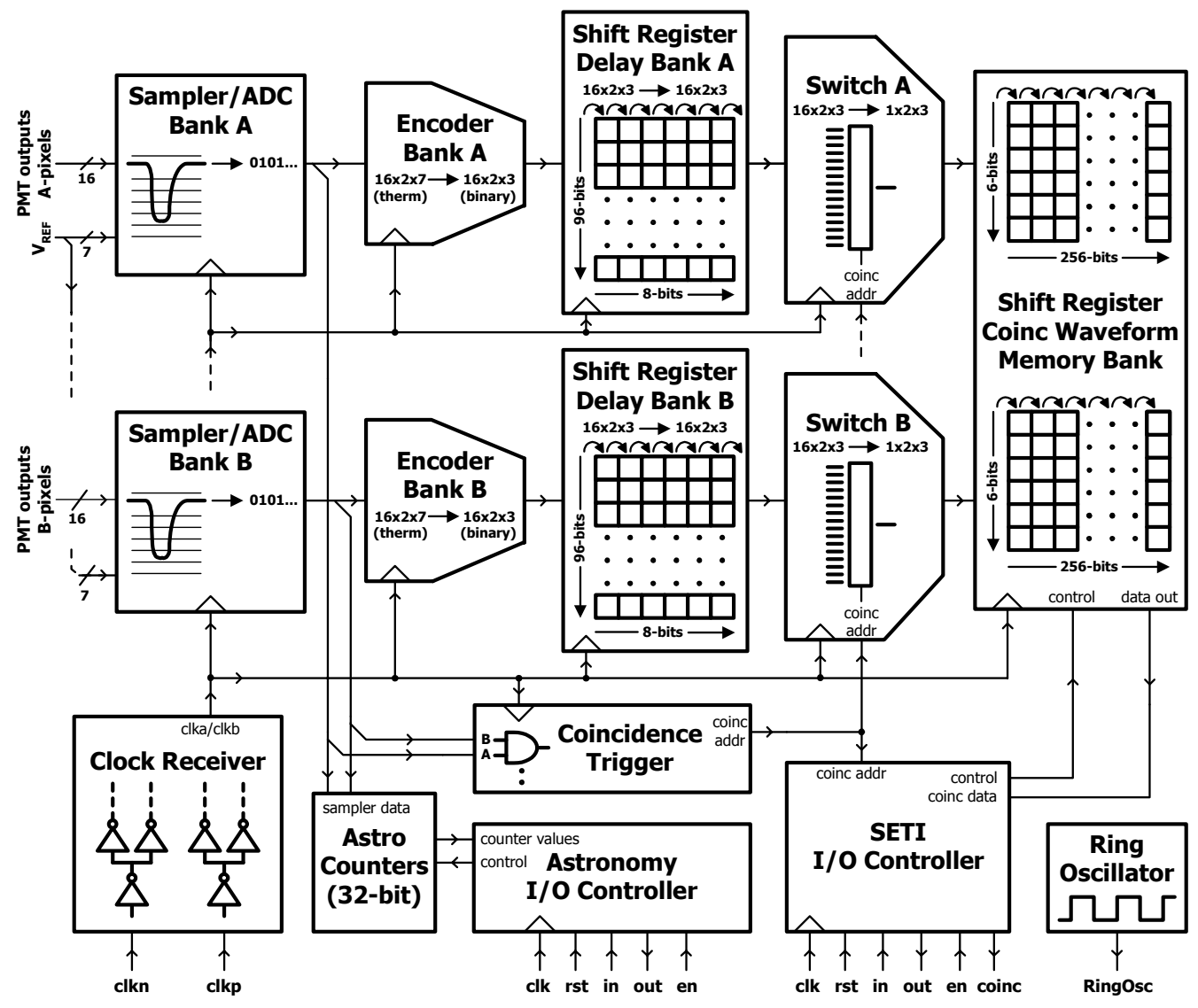

Figure 2. PulseNet top level block diagram

registers. The SETI I/O controller then passes the waveforms and coincident pixel address off chip.

PulseNet's parallel and independent function is to measure "countrates" - the number of pulses exceeding a certain $\mathrm{V}_{\mathrm{REF}}$ in a time interval. With the proper $\mathrm{V}_{\mathrm{REF}}$, this is proportional to the photon flux on the PMT pixel, i.e. it measures the brightness of the star seen by that pixel. These measurements are orchestrated by the astronomy $\mathrm{I} / \mathrm{O}$ controller, which steers streams from particular pixels and thermometer-codes, starts and stops four 32-bit counters, and transmits the data off chip.

Fig. 3 shows the sampling scheme in greater detail. An analog input $\left(\mathrm{V}_{\mathrm{PMT}}\right)$ is compared with $\mathrm{V}_{\mathrm{REF}}[6: 0]$ on the rising edges of the interleaved clka and clkb, producing the 7-bit thermometer code for each sample. Note that $\mathrm{V}_{\mathrm{REF}}[6: 1]$ is less than $\mathrm{V}_{\text {PMT }}$ since PMTs produce negative pulses; in order to detect $\mathrm{V}_{\text {PMT }}$ samples that exceed its bias voltage (an artifact of spurious PMT signals), we set $\mathrm{V}_{\text {REF }}[0]>\mathrm{V}_{\text {PMT }}$. Fig. 4 shows $V_{P M T}$ and $V_{\text {REF }}[6: 0]$ wired to comparators that produce the thermometer-coded samples. Fig 5 illustrates one of these comparators in greater detail; $\mathrm{V}_{\mathrm{PMT}}$ is compared to $\mathrm{V}_{\mathrm{REF}}$ on the rising edges of clka and clkb in modified StrongArm sense amplifiers (SAs), whose outputs drive a second stage of reduced-size SAs (improving gain and reducing hysteresis), followed by RS latches. The SA (see [2] and references therein) is a simple clocked, regenerative, differential amplifier shown in Fig. 6. The design provides good input isolation and a small aperture time (tens of ps) that can resolve small voltage differences (tens of $\mathrm{mV}$ ). The 448 SAs on PulseNet are grouped in four blocks with staggered clocking to mitigate power supply noise. Fig. 7 shows a measured signal reconstruction for one PMT signal.

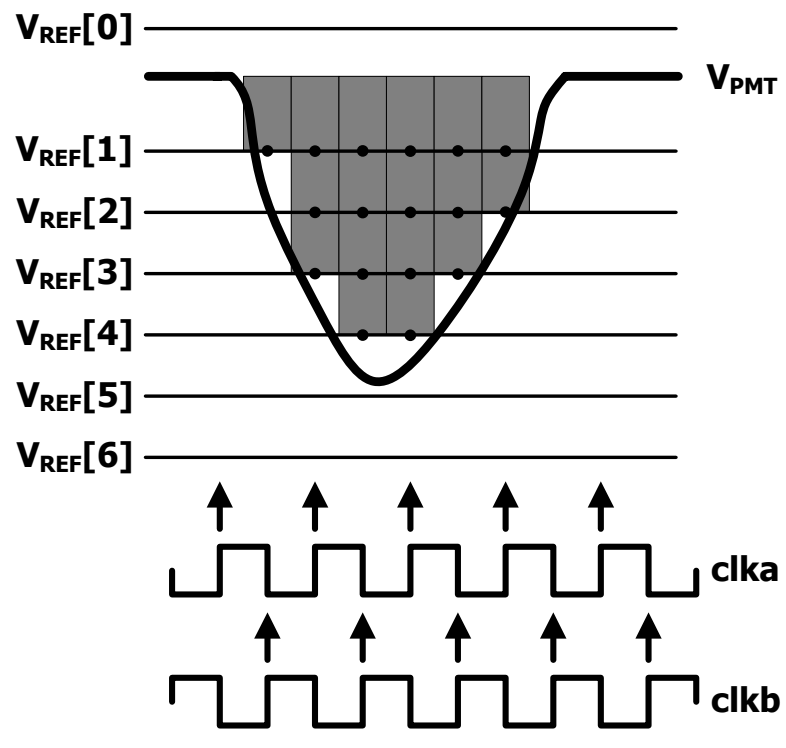

Figure 3. PMT signal sampling scheme 


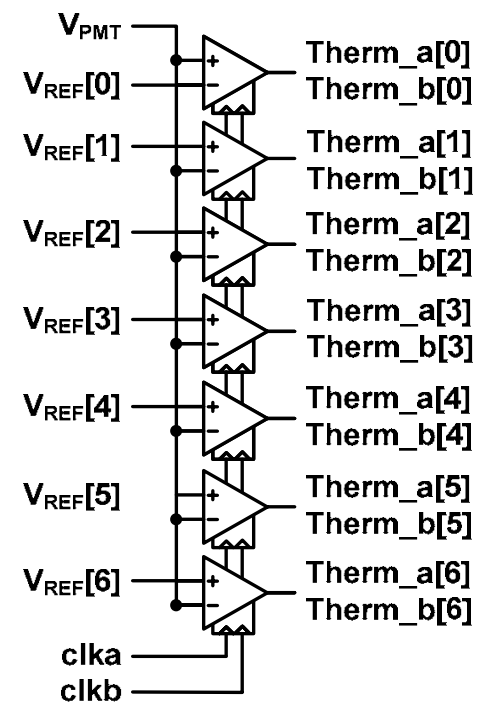

Figure 4. Thermometer-coded sampler bank.

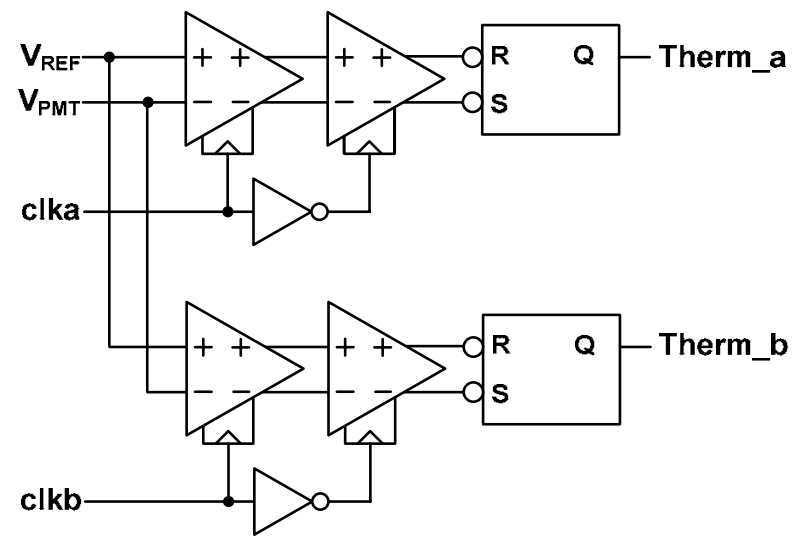

Figure 5. Detailed sampler path.

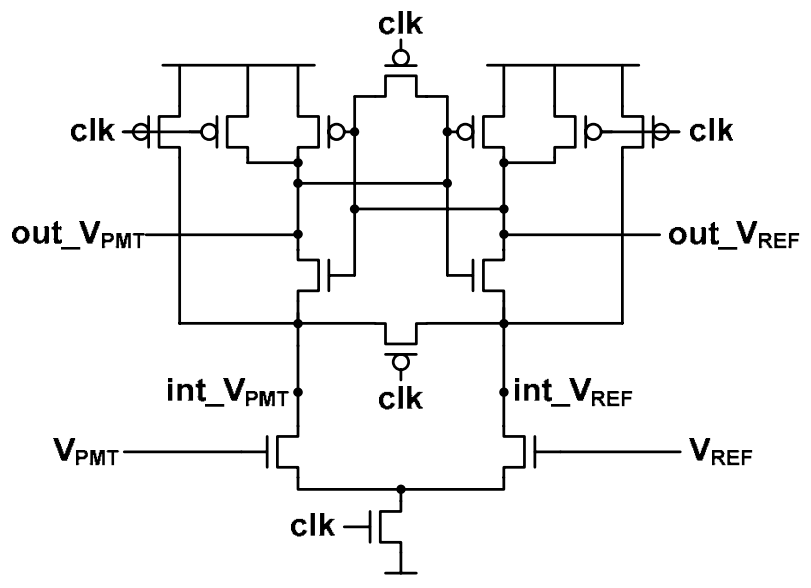

Figure 6. Modified StrongArm sense amplifier [2].

\section{SAMPler OfFset Voltage Measurements}

PulseNet's sensitivity depends on variations in input sampler offset voltage (among other things). We measured this offset on 42 chips (448 SAs per chip) and found a 1- $\sigma$ variation of $12 \mathrm{mV}$. This is consistent with the $\sim 15 \mathrm{mV}$ offset for the SA input pair predicted by the model in [3]. The full offset distribution is shown in Fig. 8; note the narrower distribution resulting from calibrating out pixel-dependent off-chip static offsets in the test setup. Fig. 9 shows offset voltage variation away from the nominal parameters used in Fig. 8: supply voltage $=2.5 \mathrm{~V}$ and input bias voltage $=1.5 \mathrm{~V}$.

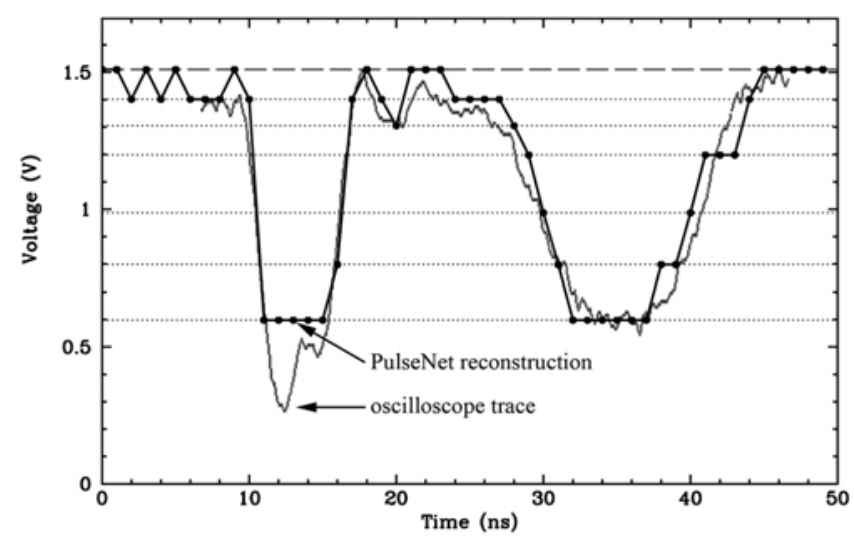

Figure 7. Waveform reconstruction of one of two analog channels (first 46 of 256 samples), sampled at $1 \mathrm{GS} / \mathrm{s}$, with oscilloscope trace for comparison.

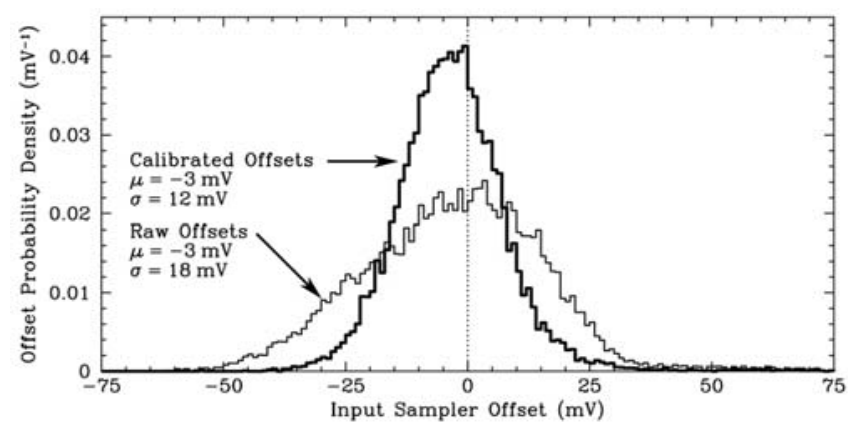

Figure 8. Histograms of input offset voltages for 42 chips (448 SAs). Note the narrower distribution after test setup calibration.

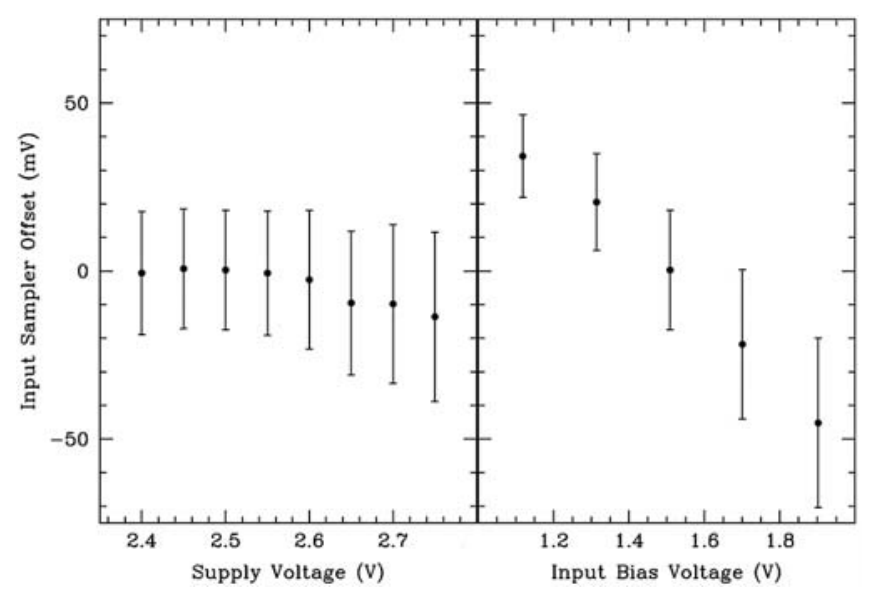

Figure 9. Offset voltage vs. $V_{\mathrm{dd}}$ and input bias. Each point is the mean, and the error bars are the 1- $\sigma$ variation, for 448 SAs on one chip. 


\section{PulseNet}

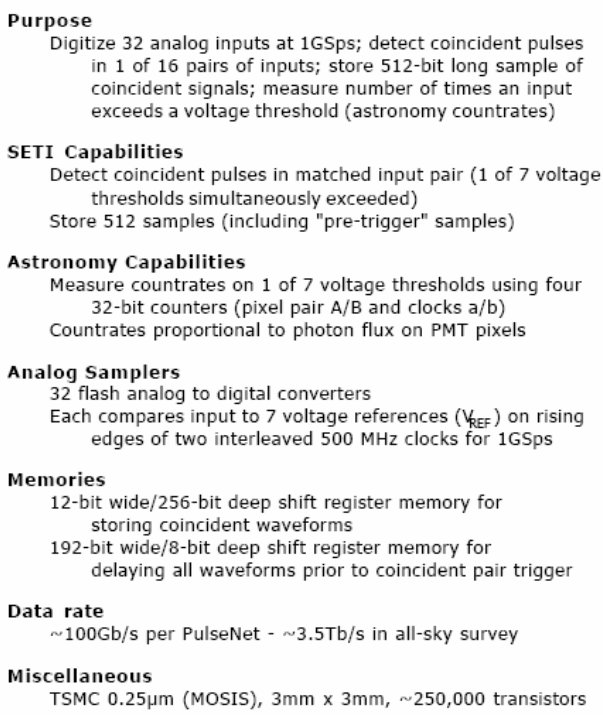

\section{All-sky Optical SETI}

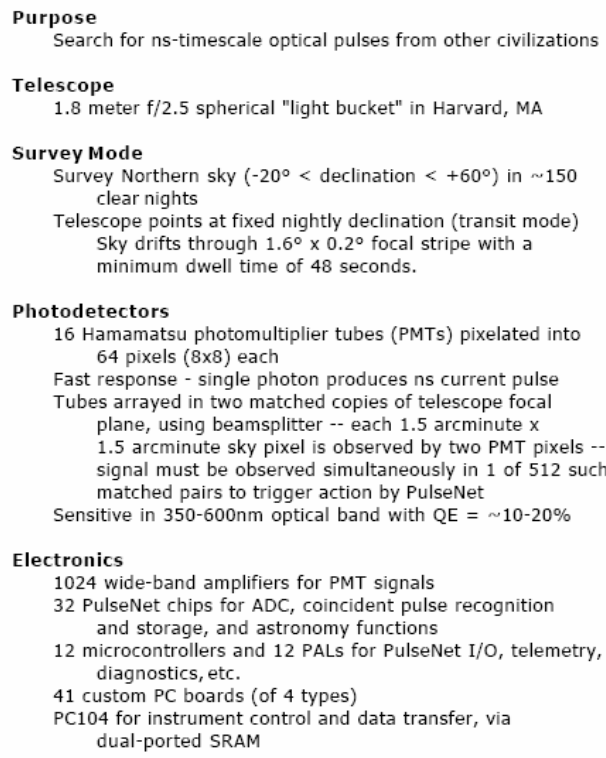

Figure 11 PulsetNet and All-sky optical SETI - table of characteristics

\section{PERFORMANCE}

PulseNet contains $\sim 250,000$ transistors, is $3.1 \mathrm{~mm} \mathrm{x}$ $3.1 \mathrm{~mm}$, and was fabricated through MOSIS on TSMC's $0.25 \mu \mathrm{m}$ CMOS process. Fig. 10 shows a die photo and gives a performance summary. All circuits were full-custom designs, with the exception of the three synthesized state machines. At $400 \mathrm{MHz}$ and $2.5 \mathrm{~V}$ (standard operation) PulseNet dissipates $1.1 \mathrm{~W}$, but has been shown to work as high as $500 \mathrm{MHz}$ and $2.87 \mathrm{~V}$. Fig. 11 presents an overall summary of characteristics of both PulseNet and All-sky optical SETI. PulseNet is fully functional and is being deployed in the all-sky optical SETI experiment as a part of the camera system shown in Fig. 12. We should see "first light" in April 2006.

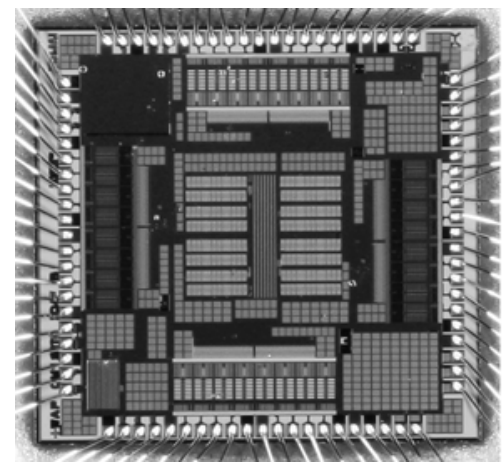

\section{PulseNet}

Purpose:

Enable all-sky optical SETI experimen Digitize 32 analog inputs at $1 \mathrm{GS} / \mathrm{s}$ Detect coincident voltage pulses Store 512-bit long coincident waveforms Measure input pulse count rates Analog samplers: 32 flash ADC (7-level) Uses StrongArm sense amplifier Offset voltage: $\sigma=12 \mathrm{mV}$ Process/size/speed: TSMC $0.25 \mu \mathrm{m}$ (MOSIS) - $2.5 \mathrm{~V}$ supply $3 \mathrm{~mm} \times 3 \mathrm{~mm}-\sim 250,000$ transistors . $\mathrm{GS} / \mathrm{s} @ 500 \mathrm{MHz}$ (interleaved clocks)

Figure 10. PulseNet die photograph and performance summary.

\section{ACKNOWLEDGEMENTS}

Authors thank members of the last three authors' groups, W. Yang, and D. Liu for discussions and circuit ideas, as well as the MOSIS Educational Program, The Bosack-Kruger Charitable Foundation, and The Planetary Society for support.

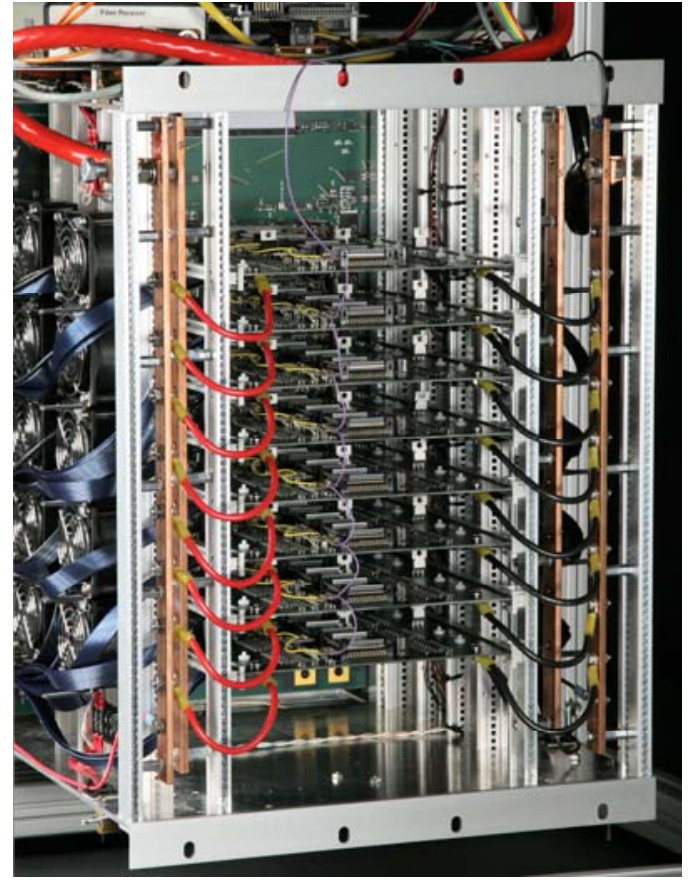

Figure 12. Camera system with 32 PulseNet chips

\section{REFERENCES}

[1] A. W. Howard et al., "Search for nanosecond optical pulses from nearby solar-type stars", Astrophysical Journal, Vol. 613, pp. 12701284, 2004

[2] M.-J. E. Lee, W. J. Dally, P. Chiang, "Low-power area-efficient highspeed I/O circuit techniques," IEEE J. Solid-State Circuits, Vol. 35, pp. 1591-1599, 2000 .

[3] M. Pelgrom, A. Duinmaijer, and A. Welbers, "Matching properties of MOS transistors," IEEE J. Solid-State Circuits, Vol. 24, pp. 1433$1440,1989$. 